\title{
Preparation and characterization of luteinising-hormone releasing hormone nanoliposomal microbubbles specifically targeting ovarian cancer cells in vitro
}

\author{
JINYI ZHANG, SISUN LIU, YUANFANG ZHU, LIPING ZHANG, \\ WENJUAN LI, FEN WANG and SHUYING HUANG \\ Department of Obstetrics and Gynaecology, The First Affiliated Hospital of Nanchang University, \\ Nanchang, Jiangxi 330006, P.R. China
}

Received July 5, 2013; Accepted March 25, 2014

DOI: $10.3892 / \mathrm{mmr} .2014 .2211$

\begin{abstract}
The aim of the present study was to prepare luteinizing-hormone releasing hormone (LHRH) nanoliposomal microbubbles specifically targeting ovarian cancer cells. The lyophilization/sonication method was used to prepare non-targeting nanoliposomal microbubbles (N-N-Mbs). Using the biotin-avidin bridge method, conjugated LHRH antibodies to N-N-Mbs generated LHRH nanoliposomal microbubbles (LHRH-N-Mbs) specifically targeting ovarian cancer cells. The morphology and physicochemical properties of the microbubbles was detected using an optical microscope and zeta detector. The binding affinity between the secondary antibody and LHRH-N-Mbs or N-N-Mbs was determined by flow cytometry. The binding of LHRH-N-Mb to human ovarian cancer cells (OVCAR-3) was detected by light microscopy. The rounded and uniformly distributed N-N-Mbs and LHRH-N-Mbs were successfully generated. The particle size ranged from 295-468 nm with a mean of $360 \mathrm{~nm}$ for N-N-Mbs or 369-618 nm with a mean of $508 \mathrm{~nm}$ for LHRH-N-Mbs. There was a significant difference in size between the two groups $(\mathrm{P}<0.05)$, although the surface potential of the two microbubbles remained the same $(-14.6 \mathrm{mV})$. Following being kept at room temperature for 14 days, no significant difference in the physicochemical properties of the LHRH-N-Mbs was detected compared with that of freshly prepared microbubbles. The secondary antibody binding rate of LHRH-N-Mbs and $\mathrm{N}-\mathrm{N}-\mathrm{Mbs}$ was 75.6 and $0.83 \%$, respectively. Furthermore, the formation of a rosette-like structure surrounding OVCAR-3 cells was observed after the cells were incubated with
\end{abstract}

Correspondence to: Dr Yuanfang Zhu, Department of Obstetrics and Gynaecology, The First Affiliated Hospital of Nanchang University, 17 Yongwaizheng Street, Nanchang, Jiangxi 330006, P.R. China

E-mail: zhuyf1027@163.com

Key words: luteinizing-hormone releasing hormone, nanoliposomal, microbubble, ultrasound contrast agent, ovarian cancer
LHRH-N-Mbs, whereas pre-incubation with LHRH antibody blocked this rosette formation. In conclusion, LHRH-N-Mbs specifically targeting ovarian cancer cells were successfully prepared through biotin-avidin mediation and the lyophilization/sonication method. The key feature of LHRH-N-Mbs is their small size, stability and high efficiency in targeting human OVCAR-3 cells in vitro.

\section{Introduction}

Ovarian cancer is the leading cause of mortality compared with all other gynecological malignancies $(1,2)$. Ovarian cancer is difficult to be diagnosed at the early stages. Consequently, $80 \%$ of patients are already at the intermediate or late stages of ovarian cancer when they present with symptoms. Qualitative and location diagnosis at the early stages of cancer progression is one of the key factors important in improving the survival rate of patients $(3,4)$. Currently, the serum biomarkers of cancer antigen-125 (CA-125) and recently identified human epididymis secretory protein-4 (HE4) are used as predictors for ovarian cancer diagnosis (5-9). The low specificity of CA-125 and HE4, however, limits their clinical application $(10,11)$. With the development of ultrasonic contrast technology, scientists have begun to consider the possibility of applying non-invasive ultrasonic molecular imaging technology (12-14) in the diagnosis of early stage ovarian cancer. The most critical part of this technique is to prepare a nano-scale targeting microbubble contrast agent. Compared with a micro-scale contrast agent, a nano-scale contrast agent has the ability to penetrate blood vessels and to be visualized by confocal imaging $(15,16)$. Low echo reflecting nano-scale contrast agents are able to penetrate the blood vessels, accumulate around the lesion and generate a significantly enhanced signal in the target area with an extremely low background signal (17). Non-specific microbubble contrast agents do not specifically interact with lesion tissues, which makes these agents unsuitable to be used for targeting. Once in the human body, the majority of microbubbles non-specifically bind to the hepatic sinusoid, the spleen sinus and the vascular endothelial system (18), so that the microbubbles are trapped in the microcirculatory system and cannot effectively reside in the 
targeted tissue for a sufficient amount of time, compromising the contrast signal. For efficient targeting in in vivo imaging, it is crucial to develop microbubbles that specifically accumulate and adhere to tumor lesions following injection into the human body.

With the progress in research on tumor-specific antigens and receptor biology, ultrasonic contrast agent targeting of malignant tumors by conjugating tumor-specific antibodies or ligands onto the surface of microbubbles has generated tumor-specific targeting microbubbles $(19,20)$. In addition, previous studies suggested that the luteinizing-hormone releasing hormone $(\mathrm{LHRH})$ receptor is overexpressed in ovarian cancer cells, with little to no expression in normal tissues $(21,22)$. Based on this evidence, it was hypothesized that by linking LHRH antibodies onto the surface of the nano-microbubble, LHRH nanoliposomal ultrasonic contrast agents targeting ovarian cancer can be prepared (Fig. 1) (23). Following penetration of the nano-scale contrast agents into the blood vessels and reaching of their target site, the LHRH antibodies on the surface of microbubbles bind to LHRH receptors in ovarian cancer cells, which results in selective accumulation. This allows for long resident time in ovarian cancer tissue and targeted ovarian cancer imaging at the molecular level by the confocal principle. The present study demonstrated the preparation of LHRH nanoliposomal microbubbles (LHRH-N-Mbs) as ultrasound contrast agents and their in vitro targeting ability of ovarian cancer cells, which provided experimental evidence for specific ultrasound imaging of ovarian cancer at an early stage.

\section{Materials and methods}

Materials. Dipalmitoyl phosphatidylcholine (DPPC) and biotinylated dipalmitoyl phosphatidylethanolamine (DSPE-PEG2000-Biotin) were ordered from Avanti Polar Lipids Inc. (Alabaster, AL, USA). Human ovarian cancer cells (OVCAR-3), McCoy's 5A medium and avidin were purchased from Wuhan Boster Biological Technology, Ltd. (Wuhan, China). Biotinylated LHRH antibody and rhodamine goat anti-rabbit immunoglobulin ( Ig) $\mathrm{G}$ were obtained from Beijing Biosynthesis Biotechnology Co., Ltd. (Beijing, China). Perfluorinated propane $\left(\mathrm{C}_{3} \mathrm{~F}_{8}\right)$ was purchased from the Medical Ultrasound Imaging Research Institute, Chongqing University (Chongqing, China).

Cell culture. The OVCAR-3 cells were cultured in McCoy's 5A medium containing $10 \%$ heat-inactivated fetal calf serum at $37^{\circ} \mathrm{C}$ and incubated in $5 \% \mathrm{CO}_{2}$. Cells were split every 4-6 days and experimental cells were in the log-growth phase.

Preparation of non-targeting nanoliposomal microbubbles $(N-N-M b s)$. DPPC and DSPE-PEG-Biotin were mixed in a $5 \mathrm{ml}$ plastic tube to form a suspension. Following lyophilization, $1 \mathrm{ml}$ of hydration solution [glycerin, phosphate-buffered saline (PBS)] was added to the samples to rehydrate them and then $\mathrm{C}_{3} \mathrm{~F}_{8}$ gas was slowly injected into the container to replace the air. Samples were then agitated using a horizontal reciprocating ultrasonic mechanical vibrator for $90 \mathrm{sec}$ to form a milky white solution. Following separation at $4{ }^{\circ} \mathrm{C}$, the bottom layer was discarded and the milky white upper layer was collected

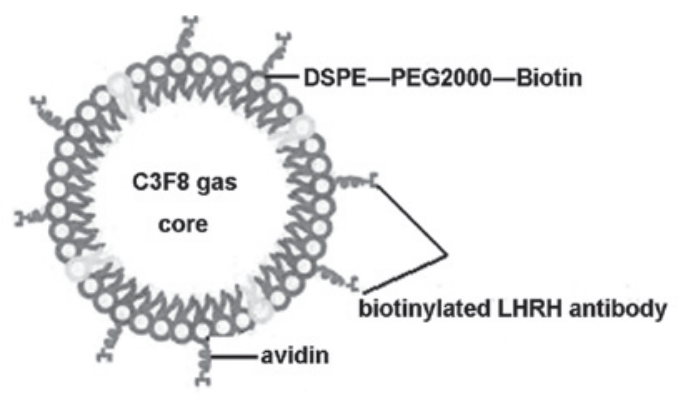

Figure 1. Schematic diagram of LHRH-N-Mb targeting ovarian cancer. The interaction between biotin and avidin is highly specific. LHRH antibodies on the surface of microbubbles bind to LHRH receptors in ovarian cancer cells, which resulted in selective accumulation, long resident time in ovarian cancer tissue and targeting of ovarian cancer. LHRH luteinizing-hormone releasing hormone; LHRH-N-Mb, LHRH nanoliposomal microbubble.

following washing with PBS three times. The upper layer was then added to $1 \mathrm{ml}$ of PBS to obtain non-targeting nanoliposomal microbubbles. Following radiation sterilization by ${ }^{60} \mathrm{Cog} \gamma$-ray (Sinotex CX, Shanghai, China), microbubbles were observed by optical microscopy to detect the morphology and particle distribution. Particle size range and the surface potential were measured by a zeta detector (Zeta sizer 3000HSA; Malvern Instruments Ltd., Worcestershire, England).

Preparation of LHRH-N-Mbs. The prepared N-N-Mbs $\left(100 \mu \mathrm{l} ; 1 \times 10^{8} / \mathrm{ml}\right)$ were mixed with saturated biotin $(100 \mu \mathrm{g})$ in an ultrasonic agitating reaction for $30 \mathrm{~min}$. Following centrifugation at $50 \mathrm{x} \mathrm{g}$ for $5 \mathrm{~min}$, the bottom layer was discarded. The upper layer was washed with PBS three times and then collected as LHRH-N-Mbs. Following sterilization, the same methods were applied to assess the physicochemical properties of the LHRH-N-Mbs.

Determination of LHRH binding onto the LHRH-N-Mb surface. Rhodamine-conjugated secondary antibodies $(1: 100 \mathrm{w} / \mathrm{v})$ were added to $100 \mu \mathrm{l}$ LHRH-N-Mb or $100 \mu \mathrm{l}$ of $\mathrm{N}-\mathrm{N}-\mathrm{Mb}$ suspension containing avidin. The two groups were incubated at $37^{\circ} \mathrm{C}$ for $30 \mathrm{~min}$, washed and observed under a fluorescence microscope (CKX41; Olympus, Tokyo, Japan) or analyzed by flow cytometry (Beckman Coulter XL; Beckman Coulter, Miami, FL, USA) to assess the binding rate.

In vitro targeting experiment. OVCAR-3 cells $\left(5 \times 10^{4} / \mathrm{ml}\right)$ were incubated with LHRH-N-Mb $\left(1 \times 10^{8} / \mathrm{ml}\right)$ at room temperature for $30 \mathrm{~min}$ prior to washing to remove free microbubbles (final volume $5 \mathrm{ml}$ ). The binding between cells and LHRH-N-Mbs was observed under a light microscope (Olympus).

Blocking experiment. OVCAR-3 cells were pre-incubated with biotinylated LHRH antibody at room temperature $\left(20-25^{\circ} \mathrm{C}\right)$ for $30 \mathrm{~min}$ prior to the cells being incubated with LHRH-N-Mbs for another $30 \mathrm{~min}$. The binding between cells and LHRH-N-Mbs was observed using light microscopy.

Statistical analysis. Values are expressed as the mean \pm standard deviation (SD) and analyzed by SAS 10.0 statistical software. The Student's t-test was employed for the comparison 


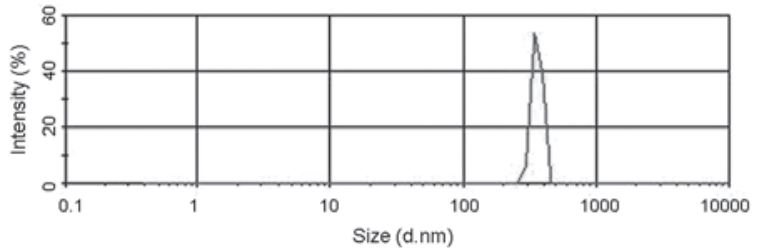

Figure 2. Physicochemical properties of N-N-Mbs. The particle size ranged from 295-468 nm with a mean of $360 \mathrm{~nm}$ and the surface potential was -14.6 mv. N-N-Mb, non-targeting nanoliposomal microbubble.

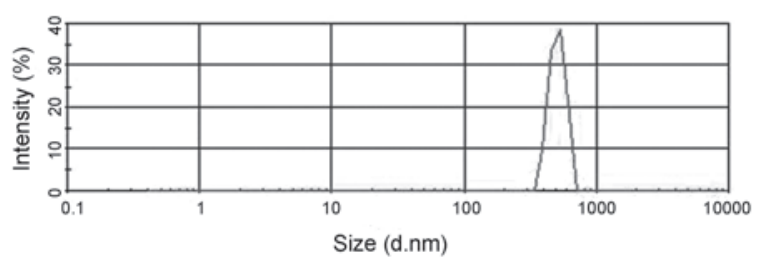

Figure 3. Physicochemical properties of LHRH-N-Mbs. The particle size ranged from 369-618 $\mathrm{nm}$ with a mean of $508 \mathrm{~nm}$ and the surface potential was -14.6 mv. LHRH-N-Mb, luteinizing-hormone releasing hormone nanoliposomal microbubble.

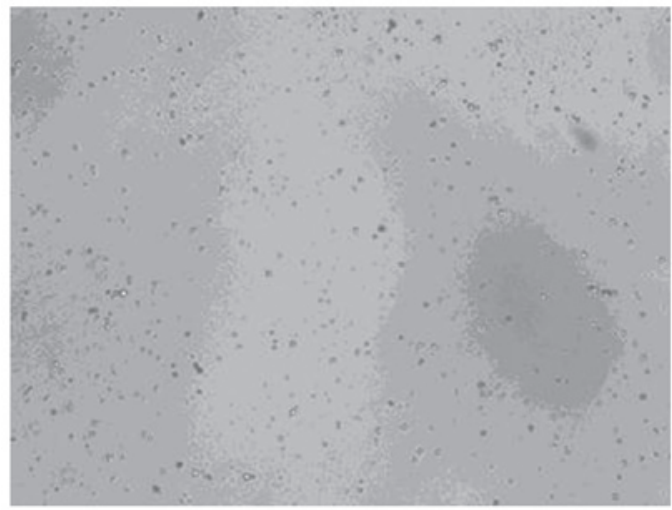

Figure 4. Under light microscopy (magnification, $\mathrm{x} 400$ ), a single LHRH-N-Mb was round following sterilization. Microbubbles were uniformly distributed with no visible aggregation. Following being kept at room temperature $\left(20-25^{\circ} \mathrm{C}\right)$ for 14 days, LHRH-N-Mb still appeared round, evenly distributed and demonstrated no signs of aggregation. LHRH-N-Mb, luteinizing-hormone releasing hormone nanoliposomal microbubble.

of two independent samples. $\mathrm{P}<0.05$ was considered to indicate a statistically significant difference.

\section{Results}

Physicochemical properties of $N-N-M b s$ and $L H R H-N-M b s$. The suspensions of the microbubbles in PBS appeared to be milky white. Light microscopy revealed that the microbubbles were uniformly distributed with no visible aggregation. A single microbubble was round following sterilization. As shown in Figs. 2 and 3, the particle size ranged from 295-468 nm with a mean of $360 \mathrm{~nm}$ for N-N-Mbs, or 369-618 nm with a mean of $508 \mathrm{~nm}$ for LHRH-N-Mbs. The surface potential in the two groups was the same $(-14.6 \mathrm{mV})$. As shown in

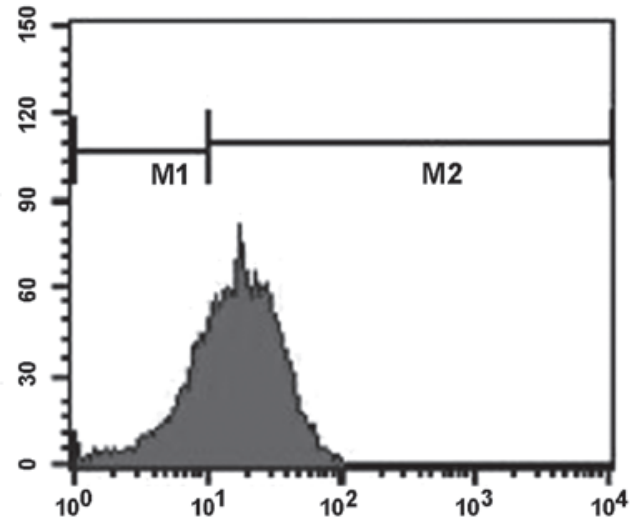

Figure 5. Flow cytometric analysis demonstrated that the binding rate between LHRH-N-Mbs and the secondary antibody was 75.6\%. LHRH-N-Mb, luteinizing-hormone releasing hormone nanoliposomal microbubble.

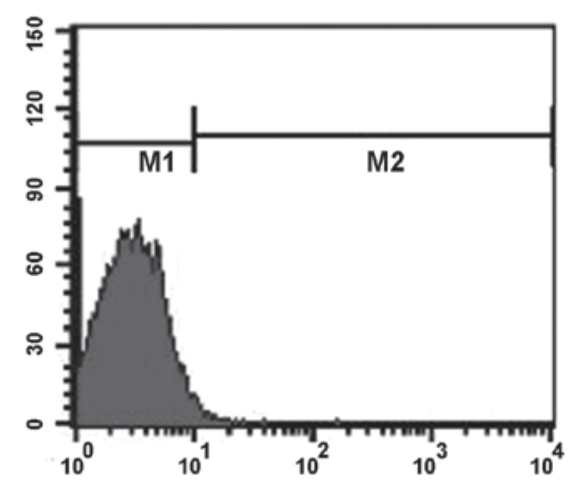

Figure 6. Flow cytometric analysis demonstrated that the binding rate between the secondary antibody and N-N-Mbs containing avidin was $0.83 \%$. $\mathrm{N}-\mathrm{N}-\mathrm{Mb}$, non-targeting nanoliposomal microbubble.

Fig. 4, following being kept at room temperature $\left(20-25^{\circ} \mathrm{C}\right)$ for 14 days, LHRH-N-Mbs appeared round, evenly distributed and showed no signs of aggregation. No significant difference was identified in particle size nor potential compared with those of freshly prepared LHRH-N-Mbs ( $\mathrm{P}>0.05)$. Following being kept at room temperature for 17 days, however, the shape of the microbubbles altered and they became uneven.

Surface LHRH binding rate of LHRH-N-Mbs N-N-Mbs bind to biotinylated LHRH antibodies via their biotin-avidin connection. Following LHRH-N-Mbs being washed four times, bright fluorescence was observed using fluorescence microscopy. The fluorescence diminished following further washing. Flow cytometry data demonstrated that the binding rate between LHRH-N-Mbs and the secondary antibody was $75.6 \%$ (Fig. 5). The binding rate between the secondary antibody and N-N-Mbs containing avidin was $0.83 \%$ (Fig. 6), which was significantly different from that between LHRH-N-Mbs and the secondary antibody $(\mathrm{P}<0.05)$. Little to no fluorescence was detected for the N-N-Mbs incubated with the secondary antibody by fluorescence microscopy.

In vitro targeting experiment. Following cells being incubated with LHRH-N-Mbs, LHRH-N-Mb was adherent to the 


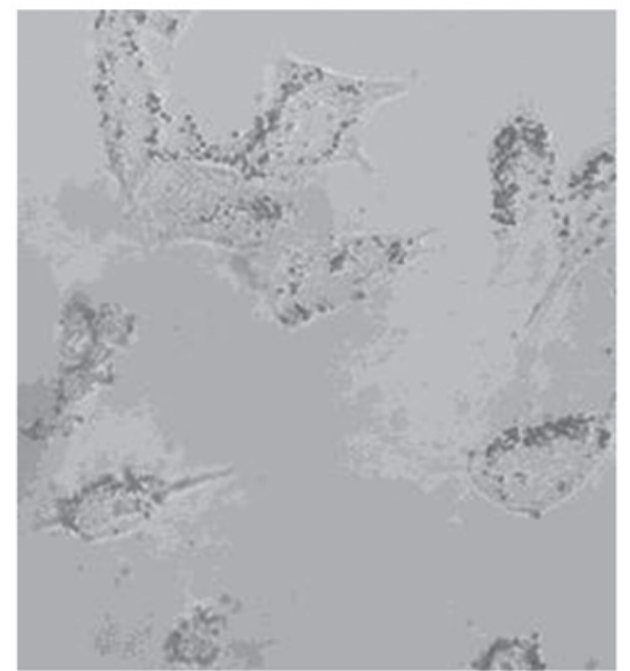

Figure 7. Light microscopy image of OVCAR-3 cells incubated with LHRH-N-Mb (magnification, x200). LHRH-N-Mb adhered to the cells and formed a rosette-like structure. LHRH-N-Mb, luteinizing-hormone releasing hormone nanoliposomal microbubble; OVCAR-3, human ovarian cancer cells.

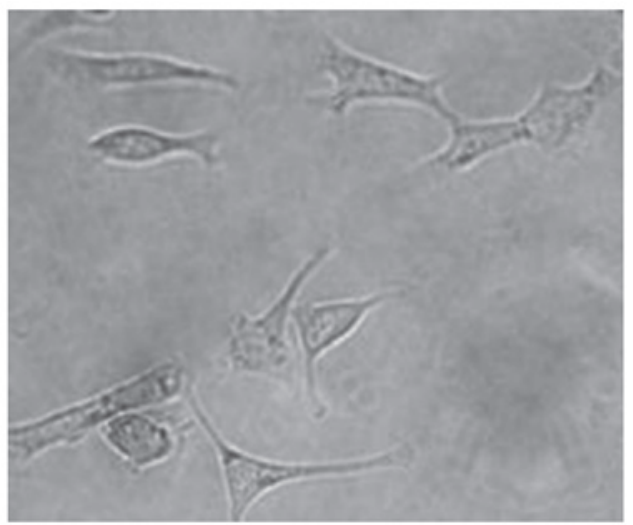

Figure 8. Light microscopy image of OVCAR-3 cells pre-incubated with LHRH antibody prior to treatment with LHRH-N-Mb. No binding of the microbubbles to OVCAR-3 cells was observed and no rosette formation was detected (magnification, x200). LHRH luteinizing-hormone releasing hormone; LHRH-N-Mb, LHRH nanoliposomal microbubble; OVCAR-3, human ovarian cancer cells.

surrounding OVCAR-3 cells and formed a rosette-like structure as observed under the light microscope (Fig. 7).

Blocking experiment. Following OVCAR-3 cells being pre-incubated with LHRH antibody, no binding between LHRH-N-Mbs and OVCAR-3 cells was observed and no rosette formation was detected (Fig. 8).

\section{Discussion}

Patients with ovarian cancer (80\%) are diagnosed at the intermediate or late stages of the malignancy $(3,4)$, which is one of the key factors contributing to the high mortality rates of ovarian cancer. Targeted ultrasound molecular imaging technology (12-14) makes early, qualitative and location diagnosis of ovarian cancer possible. In the present study, the preparation and characterization of a nano-microbubble contrast targeting agent, which is the key component of ultrasound molecular imaging, was investigated.

In the present study, N-N-Mbs and LHRH-N-Mbs for targeting ovarian cancer cells were generated using lyophilization/sonication and the biotin-avidin bridge method $(24,25)$. The particle size ranged from $295-468 \mathrm{~nm}$ with a mean of $360 \mathrm{~nm}$ for N-N-Mbs, or 369-618 nm with a mean of $508 \mathrm{~nm}$ for LHRH-N-Mbs. It was hypothesized that the biotinylated LHRH antibody may occupy a certain space, which would lead to a larger size of LHRH-N-Mbs. The in vitro targeting experiments suggested that OVCAR-3 cells highly expressing LHRH receptors efficiently bind to LHRH-N-Mbs and form a rosette-like structure. Importantly, following OVCAR-3 cells being pre-incubated with biotinylated LHRH antibodies, which blocked the interaction of receptors with its ligands, no binding of LHRH-N-Mbs to OVCAR-3 cells was observed and no rosette formation was detected, suggesting that LHRH-N-Mbs specifically bind to OVCAR-3 cells through the interaction between the biotinylated LHRH antibody and the LHRH receptor.

Whilst the size of LHRH-N-Mbs is slightly larger than that of N-N-MBS, they remain within the nanometer scale and are smaller than the vascular endothelial gap $(16,26)$. Following penetration of the nano-scale contrast agent into the blood vessels and reaching its target site, it binds to ovarian cancer cells through the LHRH receptor-antibody interaction, which leads to an extended residing time in the targeted tissue and organs. As low echo-reflecting nano-particles selectively accumulate at the target area with long residual time, nano-particles are able to produce a significantly enhanced signal at the target area and show an extremely low background noise, making LHRH-N-Mbs an ideal contrast agent at the molecular level. Adaptation of this molecular imaging technology would markedly improve the accuracy and resolution of early stage ovarian cancer diagnosis. Furthermore, the physicochemical properties of LHRH-N-Mbs did not significantly change even following being stored at room temperature for half a month, suggesting it is stable. This feature further suggests the use of this contrast agent in future in vivo experiments.

In terms of in vitro experiments, the use of LHRH-N-Mbs has less interference factors, differing from the complications of in vivo studies. The first challenge of in vivo experiments is the low concentration of the contrast agent due to the dilution in the blood. In addition, the contrast agent faces shear stress $(27,28)$ in the circulatory system, phagocytic immune response and other harsh conditions $(18,29)$. Therefore, the efficient and specific targeting of LHRH-N-Mbs in an in vivo setting, to a large extent, relies on the amount of LHRH antibodies in the LHRH-N-Mbs and the conjugative strength between the antibody and the microbubble.

The interaction between biotin and avidin is highly specific and the high affinity binding is not affected by the dilution of the reagent, which minimizes the nonspecific effect in the practical application. The binding of biotinylated antibody and avidin is quick, specific and has no effect on the antibody activity. The antibody and avidin complex is also stable as it cannot be washed off during the incubation and washing steps nor in an organic solvent $(24,25,30,31)$.

In the present study, LHRH-N-Mb was prepared by linking a biotinylated LHRH antibody to N-N-Mbs containing avidin. 
Despite repeated washing, the flow cytometry data demonstrated that the binding rate between the secondary antibody and LHRH-N-Mb was $75.6 \%$, while the binding rate between $\mathrm{N}-\mathrm{N}-\mathrm{Mb}$ containing avidin and the secondary antibody was only $0.83 \%$. As the same amount of avidin was added to LHRH-N-Mbs and N-N-Mbs, this ruled out the possibility that more binding between the secondary antibody and the microbubble was due to more avidin. These data convincingly suggest that biotinylated LHRH antibody, instead of avidin, binds to the secondary antibody. This strong interaction provides an important basis for future in vivo applications.

Numerous problems of targeting nano-microbubbles remain, including the complexity of the preparation (32), the stability in the blood and the induced immune response (33), which require further investigation. However, with improvements in technology, ultrasound molecular imaging targeting technology, a non-invasive detection method for early stage cancer diagnosis, possesses great application potential.

\section{Acknowledgements}

This study was supported by the National Natural Science Foundation of China (no. 81060217) and the Science and Technology Program Foundation of Jiangxi Province (no. 2010BSA15000).

\section{References}

1. Siegel R, Naishadham D and Jemal A: Cancer statistics, 2012. CA Cancer J Clin 62: 10-29, 2012.

2. Lech A, Daneva T, Pashova S, et al: Ovarian cancer as a genetic disease. Front Biosci (Landmark Ed) 18: 543-563, 2013.

3. Bast RC Jr, Skates S, Lokshin A and Moore RG: Differential diagnosis of a pelvic mass: improved algorithms and novel biomarkers. Int J Gynecol Cancer 22 Suppl 1: S5-S8, 2012.

4. Leung F, Dimitromanolakis A, Kobayashi H, Diamandis EP and Kulasingam V: Folate-receptor 1 (FOLR1) protein is elevated in the serum of ovarian cancer patients. Clin Biochem 46: 1462-1468, 2013.

5. Li J, Chen H, Mariani A, et al: HE4 (WFDC2) Promotes Tumor Growth in Endometrial Cancer Cell Lines. Int J Mol Sci 14: 6026-6043, 2013.

6. Ferraro S, Braga F, Lanzoni M, Boracchi P, Biganzoli EM and Panteghini M: Serum human epididymis protein 4 vs carbohydrate antigen 125 for ovarian cancer diagnosis: a systematic review. J Clin Pathol 66: 273-281, 2013.

7. Azzam AZ, Hashad DI and Kamel NA: Evaluation of HE4 as an extrabiomarker to CA125 to improve detection of ovarian carcinoma: is it time for a step forward? Arch Gynecol Obstet 288 $167-172,2013$.

8. Lenhard M, Stieber P, Hertlein L, et al: The diagnostic accuracy of two human epididymis protein 4 (HE4) testing systems in combination with CA125 in the differential diagnosis of ovarian masses. Clin Chem Lab Med 49: 2081-2088, 2011.

9. Hamed EO, Ahmed H, Sedeek OB, Mohammed AM, Abd-Alla AA and Abdel Ghaffar HM: Significance of HE4 estimation in comparison with CA125 in diagnosis of ovarian cancer and assessment of treatment response. Diagn Pathol 8: 11, 2013.

10. Varga D, Deniz M, Schwentner L and Wiesmüller L: Ovarian cancer: in search of better marker systems based on DNA repair defects. Int J Mol Sci 14: 640-673, 2013.

11. Arits AH, Stoot JE, Botterweck AA, Roumen FJ and Voogd AC Preoperative serum CA125 levels do not predict suboptimal cytoreductive surgery in epithelial ovarian cancer. Int J Gynecol Cancer 18: 621-628, 2008.
12. Deshpande N, Ren Y, Foygel K, Rosenberg J and Willmann JK: Tumor angiogenic marker expression levels during tumor growth: longitudinal assessment with molecularly targeted microbubbles and US imaging. Radiology 258: 804-811, 2011.

13. Willmann JK, Kimura RH, Deshpande N, Lutz AM, Cochran JR and Gambhir SS: Targeted contrast-enhanced ultrasound imaging of tumor angiogenesis with contrast microbubbles conjugated to integrin-binding knottin peptides. J Nucl Med 51: 433-440, 2010.

14. Barua A, Bitterman P, Bahr JM, et al: Detection of tumor-associated neoangiogenesis by Doppler ultrasonography during early-stage ovarian cancer in laying hens: a preclinical model of human spontaneous ovarian cancer. J Ultrasound Med 29: $173-182,2010$.

15. Xing Z, Wang J, Ke H, et al: The fabrication of novel nanobubble ultrasound contrast agent for potential tumor imaging. Nanotechnology 21: 145607, 2010.

16. Rapoport NY, Nam KH, Gao Z and Kennedy A: Application of Ultrasound for Targeted Nanotherapy of Malignant Tumors. Acoust Phys 55: 594-601, 2009.

17. Krupka TM, Solorio L, Wilson RE, Wu H, Azar N and Exner AA: Formulation and characterization of echogenic lipid-Pluronic nanobubbles. Mol Pharm 7: 49-59, 2010.

18. Brigger I, Dubernet C and Couvreur P: Nanoparticles in cancer therapy and diagnosis. Adv Drug Deliv Rev 54: 631-651, 2002.

19. Anderson $\mathrm{CR}, \mathrm{Hu} \mathrm{X}$, Zhang $\mathrm{H}$, et al: Ultrasound molecular imaging of tumor angiogenesis with an integrin targeted microbubble contrast agent. Invest Radiol 46: 215-224, 2011.

20. Xing W, Zhigang W, Bing H, et al: Targeting an ultrasound contrast agent to folate receptors on ovarian cancer cells: feasibility research for ultrasonic molecular imaging of tumor cells. J Ultrasound Med 29: 609-614, 2010.

21. Völker P, Gründker C, Schmidt O, Schulz KD and Emons G: Expression of receptors for luteinizing hormone-releasing hormone in human ovarian and endometrial cancers: frequency, autoregulation, and correlation with direct antiproliferative activity of luteinizing hormone-releasing hormone analogues. Am J Obstet Gynecol 186: 171-179, 2002.

22. Engel JB, Schally AV, Buchholz S, Seitz S, Emons G and Ortmann O: Targeted chemotherapy of endometrial, ovarian and breast cancers with cytotoxic analogs of luteinizing hormone-releasing hormone (LHRH). Arch Gynecol Obstet 286: 437-442, 2012.

23. Yin $\mathrm{T}$, Wang $\mathrm{P}$, Zheng $\mathrm{R}$, et al: Nanobubbles for enhanced ultrasound imaging of tumors. Int J Nanomedicine 7: 895-904, 2012.

24. Reches M and Gazit E: Biological and chemical decoration of peptide nanostructures via biotin-avidin interactions. J Nanosci Nanotechnol 7: 2239-2245, 2007.

25. Bayer EA and Wilchek M: Avidin-biotin technology: preparation of avidin conjugates. Methods Mol Biol 10: 143-148, 1992.

26. Oeffinger BE and Wheatley MA: Development and characterization of a nano-scale contrast agent. Ultrasonics 42: 343-347, 2004.

27. Saxer T, Zumbuehl A and Müller B: The use of shear stress for targeted drug delivery. Cardiovasc Res 99: 328-333, 2013.

28. Tan J, Shah S, Thomas A, Ou-Yang HD and Liu Y: The influence of size, shape and vessel geometry on nanoparticle distribution. Microfluid Nanofluidics 14: 77-87, 2013.

29. Diaz-López R, Tsapis N, Santin M, et al: The performance of PEGylated nanocapsules of perfluorooctyl bromide as an ultrasound contrast agent. Biomaterials 31: 1723-1731, 2010.

30. Morag E, Bayer EA and Wilchek M: Reversibility of biotin-binding by selective modification of tyrosine in avidin. Biochem J 316: 193-199, 1996.

31. Wang W, Liu GJ, Xie XY, et al: Development and evaluation of lipid microbubbles targeted to alpha(v)beta(3)-integrin via biotin-avidin bridge. J Microencapsul 29: 177-184, 2012.

32. Klibanov AL: Ligand-carrying gas-filled microbubbles: ultrasound contrast agents for targeted molecular imaging. Bioconjug Chem 16: 9-17, 2005.

33. Klibanov AL: Ultrasound molecular imaging with targeted microbubble contrast agents. J Nucl Cardiol 14: 876-884, 2007. 